\title{
Pattern recognition technologies for multimedia information processing
}

\author{
Sang-Soo Yeo $\cdot$ Ken Chen $\cdot$ Honghai Liu
}

Published online: 27 May 2014

(C) Springer Science+Business Media New York 2014

\begin{abstract}
Advanced algorithms based on pattern recognition for segmentation, recognition, and indexing of image, video, speech and audio data have been investigated and developed to analyze multimedia information, recently. Pattern recognition algorithms generally aim to provide a reasonable answer for all possible inputs and to perform most likely matching of the inputs, taking into account their statistical variation. Theses algorithms have been widely used in many applications to recognize what people understand such as objects, faces, human actions, and so on. Although there has been a great progress in fields of image and speech processing, virtual and augmented reality, human-computer interaction, and so on, we still have the difficulties of implementing general models that are robust to real-world variations. In this special issue, our goal is to consolidate the recent research achievements that include diverse pattern recognition based information processing for multimedia applications.
\end{abstract}

\section{Introduction}

Despite of increasing interest of researchers to issues related to pattern recognition and machine learning over the last decade, it is still imperfectly understood and studied.

We have received many manuscripts and each manuscript was blindly reviewed by at least three reviewers consisting of guest editors and external reviewers. After the first and second review processes, nine manuscripts were finally selected to be included in this special issue.

In Section 2, we present the summaries of the manuscript accepted in this issue. In the last section, we conclude the paper with some observations and future work.

S.-S. Yeo $(\bowtie)$

Mokwon University, Daejeon, Korea

e-mail: sangsooyeo@gmail.com

K. Chen

Tsinghua University, Beijing, China

e-mail: kenchen@tsinghua.edu.cn

H. Liu

University of Portsmouth, Portsmouth, UK

e-mail: honghai.liu@port.ac.uk 


\section{Related work}

The first paper entitled "Optimization of a robot-served cart capacity using the threedimensional single bin packing problem" by Khil and Lee [6], aims to optimize the amount of a dish cart capacity which a serving robot can carry at one time. It is formulated as a threedimensional finite bin packing problem (3D-BPP). The experiments are performed to demonstrate the feasibility of the proposed scheme. The empirical and theoretical analyses follow to investigate its characteristics.

The second paper entitled "A contour tracking method of large motion object using optical flow and active contour model," by Choi et al. [1] proposes a method of tracking contour of an object with larger motion and irregular shapes in image sequence in a stable manner using the optical flow and active contour model. Findings from experiments with actual videos indicate that irregular objects with large motion are easier to track.

Another paper entitled "Automatic Registration of a Virtual Experience Space with Kinect," by Soh et al. [8] proposes a new automated initialization method to replace the traditional initialization process of a virtual experience space using Kinect. This improvement can be done by estimating 6DOF of a floor plane with depth information. It shows comparable performance and offers users the opportunity to experience virtual space in a simple, low-cost manner.

The fourth paper entitled "Occlusion Detection Using Horizontally Segmented Windows for Vehicle Tracking," by Jo et al. [2] proposes a method for detection of occluded regions while tracking vehicles in visual scenes, to make the tracking robust to partial and full blocking of the target by interfering objects. The target window is segmented into several, vertical windows, and the HSV histogram distance changes over adjacent sub-windows are used to identify occluded regions. The occluded parts are then excluded in the particle-filter based object tracking framework.

Fifth paper entitled "Directional Texture Transfer for Video," by Kang et al. [5] introduces an algorithm that transfers the texture of a reference image to a target video while retaining the directionality of the target video. Texture transfer is a method that copies the texture of a reference image to a target image. The proposed algorithm maintains the temporal coherency of the transferred texture, and controls the style of the texture transfer.

Next paper entitled "Line Recognition Algorithm for 3D Polygonal Model using a Parallel Computing Platform," by Kim et al. [4] presents a method of accelerating calculation by applying CUDA (Compute Unified Device Architecture) to a line recognition algorithm that uses MLS (Moving Least Squares) surface approximation. Line recognition, however, requires a long period of calculation time and thus, various methods have been studied to accelerate the speed of the operation. The proposed technique showed that we were able to significantly reduce the time consumed in feature extraction while maintaining the quality of the model. The speed improvement algorithm suggested in the paper is not only effective for ridge-valley line but could also be effective for acceleration of other form of feature lines such as silhouette and outline, and can possibly improve the overall speed of algorithm. In this paper, we have only applied ink line effect that displays the image as if it was manually drawn by a pen. 
Seventh paper entitled "Methodology and implementation for tracking the file sharers using BitTorrent," by Park et al. [7] proposes a methodology for the investigation of illegal file sharers using BitTorrent networks through the use of a P2P digital investigation process.

Eighth paper entitled "Music Structure Analysis using Self-Similarity Matrix and Twostage Categorization," by Jun et al. [3] proposes a new method for music structure analysis on the basis of segmentation and categorization. Self-similarity matrix which is constructed by similarities among musical features is used for segmenting music. For categorizing segment, two-stage categorization method is proposed. The first stage groups segments by similar timbres and the second stage subgroups segments in each group according to chromagram sequence.

The last paper entitled "A Novel Approach to detect Network Anomalies based on IEC 61850 Substation Automation," by Yoo and Shon [9] presents proposes an anomaly detection method for substation automation based on IEC 61850. It can be used to detect the various abnormal cases in the IEC 61850 network that use the MMS and GOOSE potocols.

\section{Conclusion}

Finally, our special thanks go to Prof. Borko Furht and all editorial staffs for their valuable supports throughout the preparation and publication of this special issue. We would like to thank all authors for their contributions to this special issue. We also extend our thanks to the following external reviewers for their excellent help in reviewing the manuscripts.

\section{References}

1. Choi JW, Whangbo TK, Kim CG (2013) A contour tracking method of large motion object using optical flow and active contour model. Multimed Tools Appl. doi:10.1007/s11042-0131756-6

2. Jo A, Jang G-J, Han B (2014) Occlusion detection using horizontally segmented windows for vehicle tracking. Multimed Tools Appl. doi:10.1007/s11042-013-1846-5

3. Jun S, Rho S, Hwang E (2013) Music structure analysis using self-similarity matrix and two-stage categorization. Multimed Tools Appl. doi:10.1007/s11042-013-1761-9

4. Kang JH, Kang SJ, Kim SK (2013) Line recognition algorithm for 3D polygonal model using a parallel computing platform. Multimed Tools Appl. doi:10.1007/s11042-013-1758-4

5. Kang D, Kong P, Yoon KH, Seo SH (2013) Directional texture transfer for video. Multimed Tools Appl. doi: 10.1007/s11042-013-1759-3

6. Khil A-R, Lee K-H (2014) Optimization of a robot-served cart capacity using the three-dimensional single bin packing problem. Multimed Tools Appl. doi:10.1007/s11042-013-1843-8

7. Park S, Chung H, Lee C, Lee S, Lee K (2013) Methodology and implementation for tracking the file sharers using BitTorrent. Multimed Tools Appl. doi:10.1007/s11042-013-1760-x

8. Soh J, Han BO, Choi Y, Park Y, Seo Y-H, Yang HS (2013) Automatic registration of a virtual experience space with kinect. Multimed Tools Appl. doi:10.1007/s11042-013-1757-5

9. Yoo H, Shon T (2014) A novel approach to detect network anomalies based on IEC 61850 substation automation. Multimed Tools Appl accepted 2014 


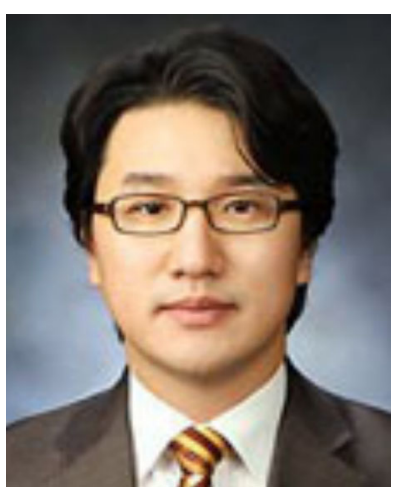

Sang-Soo Yeo received his master's degree and $\mathrm{Ph} . \mathrm{D}$ degree in Computer Science \& Engineering from ChungAng University, Seoul, Korea. He was a visiting scholar at Kyushu University, Japan. He worked for BTWorks, Inc. as a General Manager. He is currently a professor at the Division of Convergence Computer \& Media Engineering, Mokwon University, Daejeon, Korea. He is President of the Institution of Creative Research Professionals (ICRP), and Vice President of ICT Platform Society. He has been serving as chairs in many conferences and workshops. Dr. Yeo's research interests include Security, Ubiquitous Computing, Multimedia Service, Ubiquitous Computing, Embedded System, and Bioinformatics. He is a member of the KIPS, KIISE, KICS, KIISC, KMS, KDAS, KONI, KIMICS, KIIT, IEEE, IEEE CS, and IEEE ComSoc.

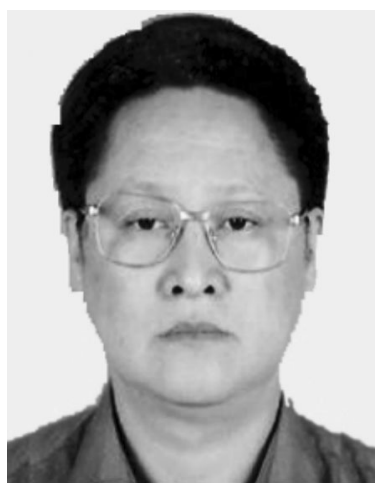

Ken Chen received the Ph.D. and M.S. degrees from Zhejiang University in 1987 and 1984 respectively, and B.S. degree from Sichuan University in 1982. From 1991 to 1992, he was a Visiting Professor at The University of Illinois at Chicago. He worked as a Postdoctoral Researcher at Purdue University at Indianapolis in 19921995. He is currently the Professor and Director in Institute of Manufacturing Engineering, Tsinghua University. His research interests are robotics and intelligent control, humanoid robots, micro and small robots, medical and space robots, manufacturing automation systems, hydraulic servo-systems. 


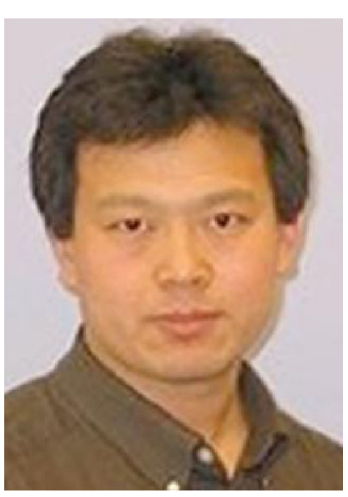

Honghai Liu received a $\mathrm{PhD}$ in intelligent robotics from King's College, University of London, currently he is Professor of Intelligent Systems and Director of Intelligent Systems and Biomedical Robotics Group, University of Portsmouth. Dr Liu is interested in approximate computation, pattern recognition, multi-sensor based information fusion and analytics, intelligent robotics and their practical applications, especially in cognitiondriven bio-mechatronics and information abstraction, with an emphasis on approaches which could make contribution to the intelligent connection of perception to action using contextual information. He is an Associate Editor of IEEE Transactions on Industrial Informatics, IEEE Transactions on Fuzzy Systems, IEEE Transactions on Systems, Man and Cybernetics, Part C, IEEE ICRA CEB and International Journal of Humanoid Robotics. $\mathrm{He}$ is also a Senior Member of IEEE and a Fellow of IET. 